\title{
GENERALIZED EULER VECTOR FIELDS ASSOCIATED TO THE WEIL BUNDLES
}

\author{
P. M. KOUOTCHOP WAMBA
}

\begin{abstract}
The notion of a Euler vector field is usually defined on the tangent bundle of a finite dimensional manifold $M$. In this paper, we generalize this notion to the Weil bundle $T^{A} M$, for any Weil algebra $A$ and we study some properties.
\end{abstract}

\section{INTRODUCTION}

Let $M$ be a smooth manifold of dimension $m \geq 1$, we denote by $T M$ the tangent bundle of $M$. Usually, an Euler vector field is defined as a vector field on $T M$ generated by the infinitesimal homotheties on the fibers of $T M$ and is denoted by $\xi_{T M}$. In local coordinate system $\left(x^{1}, \cdots, x^{m}\right)$ of $M$, we denote by $\left(x^{i}, \dot{x}^{i}\right)$ the local coordinate (adapted) of $T M$. The local expression of $\xi_{T M}$ is given by $\xi_{T M}=\dot{x}^{i} \frac{\partial}{\partial \dot{x}^{i}}$. The Euler vector field plays an essential role in the geometry of tangent bundle and is used in the global formulation of second order ordinary differential equation on a manifold $M$, it is also used to generalize to tensor fields the well known Euler's theorem on homogeneous functions. On the other hand, given a Weil algebra $A$, there is a product preserving functor $T^{A}$ from the category $\mathcal{M} f$ of all smooth manifolds and all smooth maps to $\mathcal{M f}$ which generalizes the tangent functor called Weil functor associated to $A$ (see [4]). We adopt the notations of [4] and by $T^{A} M$ we denote the smooth manifold of all $A$-velocities of $M$ and consider each element of $T^{A} M$ in the form of an $A$-jet $j^{A} \varphi, \varphi \in C^{\infty}\left(\mathbb{R}^{k}, M\right)$. By $\pi_{M}^{A}: T^{A} M \rightarrow M$ we denote the canonical projection such that, $\pi_{M}^{A}\left(j^{A} \varphi\right)=\varphi(0)$ and, for any $f \in C^{\infty}(M, N)$, the map $T^{A} f \in C^{\infty}\left(T^{A} M, T^{A} N\right)$ is defined by $T^{A} f\left(j^{A} \varphi\right)=j^{A}(f \circ \varphi)$ where $\varphi \in C^{\infty}\left(\mathbb{R}^{k}, M\right)$. When $A$ is the space of all $r$-jets of $\mathbb{R}^{k}$ into $\mathbb{R}$ with source $0 \in \mathbb{R}^{k}$ denoted by $J_{0}^{r}\left(\mathbb{R}^{k}, \mathbb{R}\right)$, the corresponding Weil functor is the functor of $k$-dimensional velocities of order $r$ and denoted by $T_{k}^{r}$, in particular for $k=1$, it is called a tangent functor of order $r$ and denoted by $T^{r}$.

The aim of this paper is to generalize the concept of Euler vector field on the Weil bundle $T^{A} M$, which will be able to be one of the mains element of the generalized Lagrangian mechanic on $T^{A} M$. We will denote it by $\xi_{T^{A} M}$. Beyond these considerations, we prove that the Euler vector field obtained is such that, for any $f \in C^{\infty}(M, N)$, the vector fields $\xi_{T^{A} M}$ and $\xi_{T^{A} N}$ are $T^{A} f$-related. In particular, we have a natural transformation

$$
\left.\xi_{A}: T^{A} \rightarrow T \circ T^{A} \quad \text { (over the identity on } T^{A}\right) .
$$

$M S C$ (2010): primary 53C15, 58A20; secondary 53C75, 53D05, 58A32.

Keywords: Weil functor, Euler vector field, Dirac structures and natural transformations. 
We define a natural Euler vector field associated to the Weil functor $T^{A}$ as a natural transformation $\xi: T^{A} \rightarrow T \circ T^{A}$ (over the identity on $T^{A}$ ) and prove that there is a canonical bijective correspondence between the set of all natural Euler vector fields $T^{A} \rightarrow T T^{A}$ and the set of all derivations of $A$. In the particular case where $A=J_{0}^{r}(\mathbb{R}, \mathbb{R})$, we prove that any natural Euler vector field $\xi: T^{r} \rightarrow T \circ T^{r}$ is of the form

$$
\sum_{\beta=1}^{r} a_{\beta} \xi_{\beta}
$$

where $a_{1}, \cdots, a_{r}$ are the real numbers and $\xi_{\beta}(1 \leq \beta \leq r)$ is the natural Euler vector field generated by the derivation $\phi_{\beta}$ on $J_{0}^{r}(\mathbb{R}, \mathbb{R}) \simeq \mathbb{R}^{r+1}$ defined below.

So, the paper is organized as follows. In Section 2 , we recall briefly the main result of [3], about lifts of tensor fields to the Weil bundle. In Section 3, we define the generalized Euler vector field on $T^{A} M$ and establish some properties. In Section 4, the concept of homogeneous tensor fields on the manifold $T^{A} M$ is defined and some properties are studied. In the last Section some homogenity properties of the tangent lifts of order $r$ of Poisson and Dirac manifolds related to Euler vector field $\xi_{T^{r} M}$ are established which generalize the similar results established in [8] and [9].

All manifolds and maps considered in the paper are assumed to be infinitely differentiable. We fix a Weil algebra $A$ of height $h \geq 2$ and of width $k \geq 1$, for any $g \in C^{\infty}\left(\mathbb{R}^{k}, \mathbb{R}\right)$ and any multiindex $\beta=\left(\beta_{1}, \cdots, \beta_{k}\right)$, we denote by

$$
D_{\beta}(g)(z)=\frac{1}{\beta !} \frac{\partial^{|\beta|} g}{\left(\partial z_{1}\right)^{\beta_{1}} \cdots\left(\partial z_{k}\right)^{\beta_{k}}}(z)
$$

the partial derivative with respect to the multiindex $\beta$ of $g$.

\section{Preliminaries}

Let $A$ be a Weil algebra, it is a real commutative and finite dimensional algebra with unit which is of the form $A=\mathbb{R} \cdot 1_{A} \oplus N_{A}$, where $N_{A}$ is the ideal of nilpotent elements of $A$. For any multiindex $\alpha \neq 0$, the vector $e_{\alpha}=j^{A}\left(x^{\alpha}\right)$ is an element of $N_{A}$ and the family $\left\{e_{\alpha}, 0<|\alpha| \leq h\right\}$ generates $N_{A}$. We denote by $B_{A}$ the set of all multiindices such that $\left(e_{\alpha}\right)_{\alpha \in B_{A}}$ is a basis of $N_{A}$ and $\bar{B}_{A}$ its complementary with respect to the set $\left\{\gamma \in \mathbb{N}^{n}, 1 \leq|\gamma| \leq h\right\}$. We put $e_{0}=1_{A}$, it is clear that $\left(e_{0}, e_{\alpha}\right)_{\alpha \in B_{A}}$ is a basis of $A$. For $\beta \in \bar{B}_{A}$, we put $e_{\beta}=\lambda_{\beta}^{\alpha} e_{\alpha}$ and we have $|\alpha|=|\beta|$ or $\lambda_{\beta}^{\alpha}=0$. So,

$$
e_{\alpha} \cdot e_{\beta}=\left\{\begin{array}{lll}
e_{\alpha+\beta} & \text { if } & \alpha+\beta \in B_{A} \\
\lambda_{\alpha+\beta}^{\gamma} e_{\gamma} & \text { if } & \alpha+\beta \in \bar{B}_{A}
\end{array} .\right.
$$

Using this basis of $A$, one deduces an adapted local coordinate system of $T^{A} M$ in the following way: let $\left(U, x^{i}\right)$ be a local coordinate system of $M$, an adapted local coordinate system induced by $\left(U, x^{i}\right)$ over the open $T^{A} U$ of $T^{A} M$ denoted 
by $\left(x_{0}^{i}, x_{\alpha}^{i}\right)_{\alpha \in B_{A}}$ is given by

$$
\left\{\begin{array}{l}
x_{0}^{i}=x^{i} \circ \pi_{M}^{A} \\
x_{\alpha}^{i}=\bar{x}_{\alpha}^{i}+\sum_{\beta \in \bar{B}_{A}} \lambda_{\beta}^{\alpha} \bar{x}_{\beta}^{i}, \text { where } \bar{x}_{\beta}^{i}\left(j^{A} g\right)=\left.\frac{1}{\beta !} \cdot D_{\beta}\left(x^{i} \circ g\right)(z)\right|_{z=0} .
\end{array}\right.
$$

In the sequel, the coordinate function $x_{0}^{i}$ is denoted by $x^{i}$. The upper index $(\alpha)$ on the tensor fields $\varphi$ on $M$ is the $\alpha$-lift of $\varphi$ to its Weil bundle (see [2,9]). More precisely, for $f \in C^{\infty}(M)$ and $\alpha \in \mathbb{N}^{n}$ such that $0 \leq|\alpha| \leq h$, we define the map $f^{(\alpha)} \in C^{\infty}\left(T^{A} M\right)(\alpha$-lift of $f)$ in the following way

$$
f^{(\alpha)}\left(j^{A} \varphi\right)=\left.\frac{1}{\alpha !} D_{\alpha}(f \circ \varphi)(z)\right|_{z=0}
$$

for any $j^{A} \varphi \in T^{A} M$. In the same way for $X \in \mathfrak{X}(M), X^{(\alpha)} \in \mathfrak{X}\left(T^{A} M\right)$ denote the $\alpha$-lift of $X$. In local coordinate $\left(x^{1}, \cdots, x^{m}\right)$ such that $X=X^{i} \frac{\partial}{\partial x^{i}}$, we have

$$
X^{(\alpha)}=\sum_{\beta \in B_{A}}\left(\left(X^{i}\right)^{(\beta-\alpha)}+\sum_{\gamma \in \overline{B_{A}}} \lambda_{\gamma}^{\beta}\left(X^{i}\right)^{(\gamma-\alpha)}\right) \frac{\partial}{\partial x_{\beta}^{i}} .
$$

For the measures of convenience, we put $f^{(\alpha)}=0$ and $X^{(\alpha)}=0$ for $|\alpha|>h$ or $\alpha \notin \mathbb{N}^{k}$.

Proposition 2.1. For any tensor field $\boldsymbol{t}$ of type $(0, p)$ on $M$, the tensor field $\boldsymbol{t}^{(\alpha)}$ is the only tensor field of type $(0, p)$ on $T^{A} M$ satisfying

$$
\boldsymbol{t}^{(\alpha)}\left(X_{1}^{\left(\beta_{1}\right)}, \cdots, X_{p}^{\left(\beta_{p}\right)}\right)=\left(\boldsymbol{t}\left(X_{1}, \cdots, X_{p}\right)\right)^{(\alpha-\beta)}
$$

where $\beta=\beta_{1}+\cdots+\beta_{p}$ and $X_{1}, \cdots, X_{p} \in \mathfrak{X}(M)$.

Proof. See [3].

\section{Main Results}

We recall that, for any $t \in \mathbb{R}$

$$
\exp (t)=e^{t}=1+\sum_{p \geq 1} \frac{t^{p}}{p !}
$$

\subsection{Euler vector fields on $T^{A} M$.}

Let $M$ be a smooth manifold of dimension $m \geq 1$. For any $\varphi \in C^{\infty}\left(\mathbb{R}^{k}, M\right)$, we consider the family of smooth maps $\left\{\varphi_{t}\right\}_{t \in \mathbb{R}} \subset C^{\infty}\left(\mathbb{R}^{k}, M\right)$ such that

$$
\varphi_{t}(z)=\varphi(\exp (t) z)
$$

for any $z \in \mathbb{R}^{k}$. We consider the smooth map

$$
\begin{aligned}
\Psi_{A, M}: \mathbb{R} \times T^{A} M & \rightarrow T^{A} M \\
\left(t, j^{A} \varphi\right) & \mapsto j^{A}\left(\varphi_{t}\right)
\end{aligned} .
$$

The map $\Psi_{A, M}$ is a one parameter subgroup of a vector field, which we denote by $\xi_{T^{A} M}$.

Definition 3.1. The vector field $\xi_{T^{A} M}$ is called a generalized Euler vector field on $T^{A} M$. 
Let $\left(U, x^{i}\right)$ be a local coordinate system of $M$ and $\left(x^{i}, x_{\alpha}^{i}\right)$ the local coordinate system of $T^{A} M$ induced by $\left(U, x^{i}\right)$. We have $\left.\frac{d\left(x^{i} \circ \Psi_{A, M}\left(t, j^{A} \varphi\right)\right)}{d t}\right|_{t=0}=0$, by the same way using the equalities

$\left.\frac{d\left(x_{\alpha}^{i} \circ \Psi_{A, M}\left(t, j^{A} \varphi\right)\right)}{d t}\right|_{t=0}=|\alpha| \bar{x}_{\alpha}^{i}\left(j^{A} \varphi\right)+\sum_{\beta \in \overline{B_{A}}}|\beta| \lambda_{\beta}^{\alpha} \bar{x}_{\beta}^{i}\left(j^{A} \varphi\right)=|\alpha| x_{\alpha}^{i}\left(j^{A} \varphi\right)$,

we deduce that the local expression of $\xi_{T^{A} M}$ is given by

$$
\xi_{T^{A} M}=\sum_{\alpha \in B_{A}}|\alpha| x_{\alpha}^{i} \frac{\partial}{\partial x_{\alpha}^{i}} .
$$

Example 3.2. (1) For $A=J_{0}^{1}\left(\mathbb{R}^{k}, \mathbb{R}\right)$, we have

$$
\xi_{T_{k}^{1} M}=\sum_{|\alpha|=1} x_{\alpha}^{i} \frac{\partial}{\partial x_{\alpha}^{i}} .
$$

In particular, when $k=1$, we have

$$
\xi_{T M}=\dot{x}^{i} \frac{\partial}{\partial \dot{x}^{i}}
$$

Therefore, $\xi_{T M}$ is the classic Euler vector field on $T M$.

(2) More generally, if $A=J_{0}^{r}\left(\mathbb{R}^{k}, \mathbb{R}\right)$, for each manifold $M$, the Euler vector field on $T_{k}^{r} M$ is given by

$$
\xi_{T_{k}^{r} M}=\sum_{1 \leq|\alpha| \leq r}|\alpha| x_{\alpha}^{i} \frac{\partial}{\partial x_{\alpha}^{i}}
$$

In particular, when $k=1$, we obtain

$$
\xi_{T^{r} M}=\sum_{\alpha=1}^{r} \alpha x_{\alpha}^{i} \frac{\partial}{\partial x_{\alpha}^{i}} .
$$

Proposition 3.3. The Euler vector field $\xi_{T^{A} M}$ is the only vector field on $T^{A} M$ satisfying

$$
\xi_{T^{A} M}\left(g^{(\alpha)}\right)=|\alpha| g^{(\alpha)}
$$

for any $0 \leq|\alpha| \leq h$ and $g \in C^{\infty}(M)$.

Proof. Let $j^{A} \varphi \in T^{A} M$ and $0 \leq|\alpha| \leq h$. Then, we have

$$
\begin{aligned}
\xi_{T^{A} M}\left(g^{(\alpha)}\right)\left(j^{A} \varphi\right) & =\left.\frac{d}{d t}\left[g^{(\alpha)}\left(j^{A} \varphi_{t}\right)\right]\right|_{t=0}=\left.\frac{d}{d t}\left[\frac{1}{\alpha !} D_{\alpha}\left(g \circ \varphi_{t}\right)\right]\right|_{t=0} \\
& =\left.\frac{1}{\alpha !} \frac{d}{d t}\left[D_{\alpha}(g \circ \varphi)(0) \exp (|\alpha| t)\right]\right|_{t=0} \\
& =|\alpha|\left(\frac{1}{\alpha !} D_{\alpha}(g \circ \varphi)(0)\right)
\end{aligned}
$$

and we deduce $\xi_{T^{A} M}\left(g^{(\alpha)}\right)=|\alpha| g^{(\alpha)}$. 
Remark 3.4. In particular, when $A=\mathbb{D}$, we have the classic formulas

$$
\left\{\begin{array}{l}
\xi_{T M}\left(g^{(0)}\right)=0 \\
\xi_{T M}\left(g^{(1)}\right)=g^{(1)}
\end{array}\right.
$$

where $g^{(0)}=g \circ \pi_{M}$ and $g^{(1)}(v)=v(g)(\varsigma)$, with $v \in T_{\varsigma} M$.

Proposition 3.5. Let $X \in \mathfrak{X}(M)$, we have

$$
\left[X^{(\alpha)}, \xi_{T^{A} M}\right]=|\alpha| X^{(\alpha)}
$$

for any $0 \leq|\alpha| \leq h$.

Proof. By calculation.

Proposition 3.6. For any tensor field $\mathbf{t}$ of the type $(0, p)$ on $M$, we have

$$
\mathcal{L}_{\xi_{T} A_{M}} \boldsymbol{t}^{(\alpha)}=|\alpha| \boldsymbol{t}^{(\alpha)}
$$

for any $0 \leq|\alpha| \leq h$.

Proof. For any $X_{1}, \cdots, X_{p} \in \mathfrak{X}(M)$ and multiindices $\beta_{1}, \cdots, \beta_{p}$ we have

$$
\begin{aligned}
& \mathcal{L}_{\xi_{T^{A} A_{M}}} \boldsymbol{t}^{(\alpha)}\left(X_{1}^{\left(\beta_{1}\right)}, \cdots, X_{p}^{\left(\beta_{p}\right)}\right) \\
= & \mathcal{L}_{\xi_{T^{A} M}}\left(\boldsymbol{t}\left(X_{1}, \cdots, X_{p}\right)\right)^{(\alpha-\beta)}-\sum_{i=1}^{p} \boldsymbol{t}^{(\alpha)}\left(X_{1}^{\left(\beta_{1}\right)}, \cdots, \mathcal{L}_{\xi_{T} A_{M}} X_{i}^{\left(\beta_{i}\right)}, \cdots, X_{p}^{\left(\beta_{p}\right)}\right) \\
= & |\alpha-\beta|\left(\boldsymbol{t}\left(X_{1}, \cdots, X_{p}\right)\right)^{(\alpha-\beta)}+|\beta|\left(\boldsymbol{t}\left(X_{1}, \cdots, X_{p}\right)\right)^{(\alpha-\beta)} \\
= & |\alpha| \boldsymbol{t}^{(\alpha)}\left(X_{1}^{\left(\beta_{1}\right)}, \cdots, X_{p}^{\left(\beta_{p}\right)}\right) .
\end{aligned}
$$

So, $\mathcal{L}_{\xi_{T} A_{M}} \boldsymbol{t}^{(\alpha)}=|\alpha| \boldsymbol{t}^{(\alpha)}$.

\subsection{Natural Euler vector fields}

Let $M$ and $N$ be smooth manifolds, we begin this subsection by the fundamental property.

Proposition 3.7. For any $f \in C^{\infty}(M, N)$, the Euler vector fields $\xi_{T^{A} M}$ on $T^{A} M$ and $\xi_{T^{A} N}$ on $T^{A} N$ are $T^{A} f$-related.

Proof. Let $j^{A} \varphi \in T^{A} M$. Then we have

$$
\begin{aligned}
T T^{A} f \circ \xi_{T^{A} M}\left(j^{A} \varphi\right) & =T T^{A} f\left(\left.\frac{d}{d t} \Psi_{A, M}\left(t, j^{A} \varphi\right)\right|_{t=0}\right) \\
& =\left.\frac{d}{d t}\left[T^{A} f\left(j^{A} \varphi_{t}\right)\right]\right|_{t=0}=\left.\frac{d}{d t}\left(j^{A}\left(f \circ \varphi_{t}\right)\right)\right|_{t=0} \\
& =\left.\frac{d}{d t} \Psi_{A, M}\left(t, j^{A}(f \circ \varphi)\right)\right|_{t=0}=\xi_{T^{A} N} \circ T^{A} f\left(j^{A} \varphi\right) .
\end{aligned}
$$

Therefore, $T T^{A} f \circ \xi_{T^{A} M}=\xi_{T^{A} N} \circ T^{A} f$.

Definition 3.8. We call natural Euler vector fields associated to $T^{A}$ any natural transformation $T^{A} \rightarrow T \circ T^{A}$ over the identity of $T^{A}$.

Example 3.9. By Proposition 3.7, it follows that the family $\left\{\xi_{T^{A} M}\right\}$ is a natural transformation $T^{A} \rightarrow T \circ T^{A}$ over the identity of $T^{A}$. So, it is a natural Euler vector field associated to $T^{A}$ which we denote $\xi_{A}: T^{A} \rightarrow T \circ T^{A}$. 
Given two Weil algebras $A$ and $B$, all natural transformations $T^{A} \rightarrow T^{B}$ correspond exactly to the algebra homomorphism from $A$ to $B$. In fact, for a natural transformation $\varphi^{A, B}: T^{A} \rightarrow T^{B}$, the algebra homomorphism associated is given by the linear map

$$
\varphi_{\mathbb{R}}^{A, B}: A=T^{A} \mathbb{R} \rightarrow T^{B} \mathbb{R}=B .
$$

On the other hand, the functor $T \circ T^{A}$ corresponds to Weil algebra $\mathbb{D} \otimes A$ which is identified with the Weil algebra $A^{2}=A \times A$ endowed by the following structure: for any $(a, b),\left(a^{\prime}, b^{\prime}\right) \in A^{2}$,

$$
(a, b) \cdot\left(a^{\prime}, b^{\prime}\right)=\left(a a^{\prime}, a b^{\prime}+a^{\prime} b\right) .
$$

Proposition 3.10. Let $\varphi^{A, B}: T^{A} \rightarrow T^{B}$, the algebra homomorphism associated to Weil algebras $A$ and $B$. We have

$$
T\left(\varphi_{M}^{A, B}\right) \circ \xi_{T^{A} M}=\xi_{T^{B} M} \circ \varphi_{M}^{A, B}
$$

for any $m$-dimensional manifold $M$. In other words, the vector fields $\xi_{T^{A} M}$ and $\xi_{T^{B} M}$ are $\varphi_{M}^{A, B}$-related.

Proof. Let $j^{A} g \in T^{A} M$, we put $F_{t}=\Psi_{A, M}(t, \cdot)$. We have

$$
\begin{aligned}
T\left(\varphi_{M}^{A, B}\right) \circ \xi_{T^{A} M}\left(j^{A} g\right) & =T\left(\varphi_{M}^{A, B}\right)\left(\left.\frac{d}{d t} F_{t}\left(j^{A} g\right)\right|_{t=0}\right) \\
& =\left.\frac{d}{d t}\left(\varphi_{M}^{A, B} \circ F_{t}\left(j^{A} g\right)\right)\right|_{t=0} \\
& =\left.\frac{d}{d t}\left(F_{t} \circ \varphi_{M}^{A, B}\left(j^{A} g\right)\right)\right|_{t=0} \\
& =\xi_{T^{B} M}\left(\varphi_{M}^{A, B}\left(j^{A} g\right)\right) .
\end{aligned}
$$

Thus, we get $T\left(\varphi_{M}^{A, B}\right) \circ \xi_{T^{A} M}\left(j^{A} g\right)=\xi_{T^{B} M}\left(\varphi_{M}^{A, B}\left(j^{A} g\right)\right)$ for any $j^{A} g \in T^{A} M$.

Remark 3.11. We assume that $A$ is a $(k, r)$-algebra. The surjective algebra homomorphism $\varrho: J_{0}^{r}\left(\mathbb{R}^{k}, \mathbb{R}\right) \rightarrow A$ determines a natural transformation $\varrho: T_{k}^{r} \rightarrow$ $T^{A}$. So, for any manifold $M$ the vector fields $\xi_{T_{k}^{r} M}$ and $\xi_{T^{A} M}$ are $\varrho_{M}$-related.

Applying the theory of Weil functors, we characterize all natural Euler vector fields on Weil functors (bundles) as follows.

Theorem 3.12. There is a bijective correspondence between the set of natural Euler vector fields $T^{A} \rightarrow T \circ T^{A}$ and the set of the derivations of $A$.

Proof. Let $\varphi_{A}: T^{A} \rightarrow T \circ T^{A}$ the natural Euler vector field, the map $\varphi_{A, \mathbb{R}}:$ $A \rightarrow A \times A$ is an algebra homomorphism over $A$. It has the form

$$
\varphi_{A, \mathbb{R}}(a)=(a, D(a))
$$

where $D: A \rightarrow A$ is a linear map. On the other hand, for any $a, b \in A$,

$$
\varphi_{A, \mathbb{R}}(a b)=\varphi_{A, \mathbb{R}}(a) \cdot \varphi_{A, \mathbb{R}}(b) .
$$


Since

$$
\left\{\begin{array}{ccc}
\varphi_{A, \mathbb{R}}(a) \cdot \varphi_{A, \mathbb{R}}(b) & = & (a b, a D(b)+b D(a)) \\
\varphi_{A, \mathbb{R}}(a b) & = & (a b, D(a b))
\end{array},\right.
$$

we obtain $D(a b)=a D(b)+b D(a)$. So, $D$ is a derivation of $A$.

Inversely, consider $D: A \rightarrow A$ the derivation, the map

$$
\begin{aligned}
\varphi_{D}^{A}: A & \rightarrow A \times A \\
a & \mapsto(a, D(a))
\end{aligned}
$$

is a morphism of Weil algebras. It induces a natural transformation $\bar{\varphi}_{D}^{A}: T^{A} \rightarrow$ $T \circ T^{A}$. It is clear that $\bar{\varphi}_{D, \mathbb{R}}^{A}=\varphi_{A, \mathbb{R}}$. The rest of the proof is similar to Theorem (35.13) in [4].

Remark 3.13. Let $D: A \rightarrow A$ be a derivation, we consider the Euler vector field $\xi_{D, T^{A} M}$ induced by $D$ on a $m$-dimensional manifold $M$. In local coordinate $\left(x_{1}, \cdots, x_{m}\right)$, we have

$$
\xi_{D, T^{A} M}=\sum_{\alpha, \beta \in B_{A}}\left(e_{\beta}^{*} \circ D\left(e_{\alpha}\right)\right) x_{\alpha}^{i} \frac{\partial}{\partial x_{\alpha}^{i}}
$$

where $\left(e_{\alpha}\right)_{\alpha \in B_{A}}$ is the basis of $N_{A},\left(e_{\alpha}^{*}\right)_{\alpha \in B_{A}}$ its dual and $D\left(e_{0}\right)=0$.

Let $\varphi_{1}: T^{A} \rightarrow T \circ T^{A}$ and $\varphi_{2}: T^{A} \rightarrow T \circ T^{A}$ be two natural Euler vector fields. For any real number $b$, we define the natural Euler vector fields $\varphi_{1}+\varphi_{2}$ and $b \varphi_{1}$ by

$$
\begin{aligned}
\left(\varphi_{1}+\varphi_{2}\right)_{M} & =\varphi_{1, M}+\varphi_{2, M} \\
\left(b \varphi_{1}\right)_{M} & =b \varphi_{1, M}
\end{aligned}
$$

for any manifold $M$. Note that $\varphi_{1, M}+\varphi_{2, M}$ is the sum of the vector fields $\varphi_{1, M}$ and $\varphi_{2, M}$, while $b \varphi_{1, M}$ is the product of vector field $\varphi_{1, M}$ by the scalar $b$. We denote by $\operatorname{Der}(A)$ the vector space of all the derivations of $A$ and $\operatorname{Nev}(A)$ the space of all natural Euler vector fields. By the theorem above, we have a map

$$
\begin{aligned}
& \Phi: \operatorname{Der}(A) \rightarrow \operatorname{Nev}(A) \\
& D \quad \mapsto \quad \bar{\varphi}_{D}^{A} .
\end{aligned}
$$

So, we have

Corollary 3.14. The map $\Phi: \operatorname{Der}(A) \rightarrow \operatorname{Nev}(A)$ is an isomorphism of vector spaces.

Example 3.15. We consider the classical Euler vector field $\xi_{T M}$ defined on $T M$. The Euler vector field $\xi_{T M}$ is obtained with the help of the derivation $d$ defined on $\mathbb{D} \simeq \mathbb{R}^{2}$ such that,

$$
d(x, y)=(0, y)
$$

for any $(x, y) \in \mathbb{R}^{2}$.

Example 3.16. The natural Euler vector field $\xi_{A}: T^{A} \rightarrow T \circ T^{A}$ (Example 3.9) is determined by the derivation $D_{A}: A \rightarrow A$ defined by

$$
D_{A}\left(e_{\alpha}\right)=|\alpha| e_{\alpha}
$$

for any $0 \leq|\alpha| \leq h$. 
Example 3.17. All natural Euler vector fields associated to the tangent functor $T$ are of the form

$$
b \xi_{T M}
$$

where $b$ is a real parameter. In fact, the structure of Weil algebra $\mathbb{D}=\mathbb{R}^{2}$ is given by

$$
\left(x_{1}, y_{1}\right) \cdot\left(x_{2}, y_{2}\right)=\left(x_{1} x_{2}, x_{1} y_{2}+x_{2} y_{1}\right) .
$$

Let $\varphi_{M}: T M \rightarrow T T M$ be the Euler vector field on $T M$, it is associated to a derivation $d: \mathbb{R}^{2} \rightarrow \mathbb{R}^{2}$. It has the form

$$
d(x, y)=(0, b y)
$$

with $b \in \mathbb{R}$. It follows that the Euler vector field associated to $\varphi_{M}$ is $b \xi_{T M}$.

In the next subsection, we generalize this result of the previous example to a tangent bundle of a higher order.

\subsection{The natural Euler vector fields $T^{r} \rightarrow T \circ T^{r}$}

Using the identification $\mathbb{R}^{r+1} \simeq J_{0}^{r}(\mathbb{R}, \mathbb{R})$ with the canonical basis $\left(e_{\alpha}\right)_{0 \leq \alpha \leq r}$ such that

$$
e_{\alpha} \cdot e_{\beta}=\frac{(\alpha+\beta) !}{\alpha ! \beta !} e_{\alpha+\beta}
$$

we have

Lemma 3.18. For any $0<\beta \leq r$, the linear map $\phi_{\beta}: J_{0}^{r}(\mathbb{R}, \mathbb{R}) \rightarrow J_{0}^{r}(\mathbb{R}, \mathbb{R})$ defined by

$$
\left\{\begin{array}{c}
\phi_{\beta}\left(e_{0}\right)=0 \\
\phi_{\beta}\left(e_{\alpha+1}\right)=\frac{(\alpha+\beta) !}{\alpha ! \beta !} e_{\alpha+\beta}
\end{array}\right.
$$

is a derivation.

Proof. By calculation.

Remark 3.19. For any $\beta=1, \cdots, r$, we denote by $\xi_{\beta}: T^{r} \rightarrow T \circ T^{r}$ the natural Euler vector field related to $\phi_{\beta}$. For any manifold $M$ of dimension $m \geq 1$, we have locally

$$
\xi_{\beta, T^{r} M}=\sum_{\alpha=0}^{r-\beta} \frac{(\alpha+\beta) !}{\alpha ! \beta !} x_{\alpha+1}^{i} \frac{\partial}{\partial x_{\alpha+1}^{i}} .
$$

Lemma 3.20. Any derivation $\phi: \mathbb{R}^{r+1} \rightarrow \mathbb{R}^{r+1}$ is of the form

$$
\phi=\sum_{\beta=1}^{r} a_{\beta} \cdot \phi_{\beta}
$$

where $a_{1}, \cdots, a_{r}$ are the real numbers.

Proof. For any $\alpha=0, \cdots, r$, we have $e_{0} \cdot e_{\alpha}=e_{\alpha}$, therefore $\phi\left(e_{\alpha}\right) \cdot e_{0}+\phi\left(e_{0}\right)$. $e_{\alpha}=\phi\left(e_{\alpha}\right)$. It follows that

$$
\phi\left(e_{0}\right) \cdot e_{\alpha}=0, \quad \forall \alpha=0, \cdots, r .
$$


Thus, $\phi\left(e_{0}\right)=0$. We put

$$
\phi\left(e_{1}\right)=\sum_{\beta=0}^{r} a_{\beta} e_{\beta}
$$

with $a_{0}, a_{1}, \cdots, a_{r}$ being real numbers. Using the relation $e_{1} \cdot e_{1}=2 e_{2}$, we have

$$
\phi\left(e_{2}\right)=\phi\left(e_{1}\right) \cdot e_{1}=\sum_{\beta=0}^{r-1}(\beta+1) a_{\beta} e_{\beta+1} .
$$

In the same way, $e_{2} \cdot e_{1}=3 e_{3}$, it follows that, $3 \phi\left(e_{3}\right)=\phi\left(e_{2}\right) \cdot e_{1}+\phi\left(e_{1}\right) \cdot e_{2}$. Now

$$
\begin{aligned}
& \phi\left(e_{2}\right) \cdot e_{1}=\sum_{\beta=0}^{r-2}(\beta+1)(\beta+2) a_{\beta} e_{\beta+2}, \\
& \phi\left(e_{1}\right) \cdot e_{2}=\sum_{\beta=0}^{r-2} \frac{(\beta+1)(\beta+2)}{2} a_{\beta} e_{\beta+2} .
\end{aligned}
$$

We deduce that,

$$
\phi\left(e_{2}\right) \cdot e_{1}+\phi\left(e_{1}\right) \cdot e_{2}=\sum_{\beta=0}^{r-2} 3 \frac{(\beta+1)(\beta+2)}{2} a_{\beta} e_{\beta+2} .
$$

So,

$$
\phi\left(e_{3}\right)=\sum_{\beta=0}^{n-2} \frac{(\beta+1)(\beta+2)}{2} a_{\beta} e_{\beta+2} .
$$

Looking the at expressions of $\phi\left(e_{1}\right), \phi\left(e_{2}\right)$ and $\phi\left(e_{3}\right)$ we put

$$
\phi\left(e_{\alpha}\right)=\sum_{\beta=0}^{r-\alpha+1} \frac{(\alpha+\beta-1) !}{(\beta-1) ! \alpha !} a_{\beta} e_{\alpha+\beta-1} .
$$

By induction, using the relation $e_{\alpha} \cdot e_{1}=(\alpha+1) e_{\alpha+1}$, we obtain

$$
(\alpha+1) \phi\left(e_{\alpha+1}\right)=\phi\left(e_{\alpha}\right) \cdot e_{1}+\phi\left(e_{1}\right) \cdot e_{\alpha} .
$$

Now,

$$
\begin{aligned}
& \phi\left(e_{\alpha}\right) \cdot e_{1}=\sum_{\beta=0}^{r-\alpha+1} \frac{(\alpha+\beta-1) !}{(\beta-1) ! \alpha !} a_{\beta} e_{\alpha+\beta-1} \cdot e_{1}=\sum_{\beta=0}^{r-\alpha} \frac{(\alpha+\beta) !}{(\beta-1) ! \alpha !} a_{\beta} e_{\alpha+\beta}, \\
& \phi\left(e_{1}\right) \cdot e_{\alpha}=\sum_{\beta=0}^{r} a_{\beta} e_{\beta} \cdot e_{\alpha}=\sum_{\beta=0}^{r-\alpha} \frac{(\alpha+\beta) !}{\beta ! \alpha !} a_{\beta} e_{\alpha+\beta} .
\end{aligned}
$$

We deduce that

$$
\phi\left(e_{\alpha}\right) \cdot e_{1}+\phi\left(e_{1}\right) \cdot e_{\alpha}=\sum_{\beta=0}^{r-\alpha} \frac{(\alpha+1)(\alpha+\beta) !}{\beta ! \alpha !} a_{\beta} e_{\alpha+\beta} .
$$

Thus,

$$
\phi\left(e_{\alpha+1}\right)=\sum_{\beta=0}^{r-\alpha} \frac{(\alpha+\beta) !}{\beta ! \alpha !} a_{\beta} e_{\alpha+\beta}
$$


On the other hand, $\phi\left(e_{r}\right)=a_{0} e_{r-1}+a_{1} e_{r}$ and $e_{r} \cdot e_{1}=0$. Thus, $\phi\left(e_{r}\right) \cdot e_{1}+$ $\phi\left(e_{1}\right) \cdot e_{r}=0$. As

$$
\begin{aligned}
& \phi\left(e_{r}\right) \cdot e_{1}=r a_{0} e_{r}, \\
& \phi\left(e_{1}\right) \cdot e_{r}=a_{0} e_{r},
\end{aligned}
$$

it follows that $a_{0}=0$. So, for any $\alpha=0, \cdots, r-1$, we have

$$
\phi\left(e_{\alpha+1}\right)=\sum_{\beta=1}^{r-\alpha} a_{\beta} \frac{(\alpha+\beta) !}{\beta ! \alpha !} e_{\alpha+\beta}=\sum_{\beta=1}^{r-\alpha} a_{\beta} \phi_{\beta}\left(e_{\alpha+1}\right) .
$$

This yields the result.

Theorem 3.21. All natural Euler vector fields $T^{r} \rightarrow T \circ T^{r}$ are of the form

$$
\sum_{\beta=1}^{r} a_{\beta} \xi_{\beta}
$$

where $a_{1}, \cdots, a_{r}$ are real numbers.

Proof. Let $E: T^{r} \rightarrow T \circ T^{r}$ be a natural Euler vector field, it induces a derivation $\phi: \mathbb{R}^{r+1} \rightarrow \mathbb{R}^{r+1}$, where the structure of Weil algebra is defined above. So, by the previous lemma, there are the real numbers $a_{1}, \cdots, a_{r}$ such that

$$
\phi=\sum_{\beta=1}^{r} a_{\beta} \phi_{\beta}
$$

On the other hand, for any manifold $M$, locally we have

$$
\begin{aligned}
E_{T^{r} M} & =\sum_{\alpha, \gamma=1}^{r}\left(e_{\gamma}^{*} \circ \phi\left(e_{\alpha}\right)\right) x_{\alpha}^{i} \frac{\partial}{\partial x_{\alpha}^{i}} \\
& =\sum_{\beta=1}^{r} \sum_{\alpha, \gamma=1}^{r} a_{\beta}\left(e_{\gamma}^{*} \circ \phi_{\beta}\left(e_{\alpha}\right)\right) x_{\alpha}^{i} \frac{\partial}{\partial x_{\alpha}^{i}} \\
& =\sum_{\beta=1}^{r} a_{\beta} \xi_{\beta, T^{r} M} .
\end{aligned}
$$

Thus, we obtain $E=\sum_{\beta=1}^{r} a_{\beta} \xi_{\beta, \cdot \cdot}$

\subsection{Absolute operators seen as natural Euler vector fields}

Let $F$ be a bundle functor on the category $\mathcal{M f}$. We denote by $0_{M}$ the zero vector field on $M$.

Definition 3.22. ([4]) A natural operator $R: T \rightsquigarrow T \circ F$ is said to be an absolute operator if $R_{M} X=R_{M} 0_{M}$ for every vector field $X$ of $M$.

Let $D$ be a derivation of $A$, for any real number $t, \phi_{t}=\exp (t D) \in \operatorname{Aut}(A)$, where $\operatorname{Aut}(A)$ is the group of all automorphisms of $A$. It is a Lie subgroup of Lie group $G L(A)$. The map $\phi_{t}: A \rightarrow A$ is an automorphism of $A$ inducing a natural transformation $\phi_{t, M}: T^{A} M \rightarrow T^{A} M$. Consider the map $D(M): \mathbb{R} \times T^{A} M \rightarrow$ $T^{A} M$ such that

$$
D(M)(t, \xi)=\phi_{t, M}(\xi)
$$


for any $(t, \xi) \in \mathbb{R} \times T^{A} M$. It is one parameter subgroup of the vector field $X_{D(M)}$ : $T^{A} M \rightarrow T T^{A} M$. On the other hand, for any $f \in C^{\infty}(M, N)$ we have $T^{A} f \circ \phi_{t, M}=$ $\phi_{t, N} \circ T^{A} f$ for every $t$. It follows that $X_{D(M)}$ and $X_{D(N)}$ are $T^{A} f$-related. We get a natural Euler vector field $X_{D}: T^{A} \rightarrow T \circ T^{A}$ associated to $D$.

Remark 3.23. The constant map $X \mapsto X_{D(M)}$ for all $X \in \mathfrak{X}(T M)$ forms an absolute operator, $\operatorname{Op}(D): T \rightsquigarrow T \circ T^{A}$, which is said to be generated by $D$. In [4], it is shown that every absolute operator $R: T \rightsquigarrow T \circ T^{A}$ is of the form $R=\mathrm{Op}(D)$.

Corollary 3.24. There is bijective correspondence between the set of absolute operators and the set of natural Euler vector fields associated to $T^{A}$.

The main result on the prolongations of vector fields related to Weil bundle is given by I. Kolář ([4]). In fact, it proves that all natural operators $T \rightsquigarrow T \circ T^{A}$ are of the form

$$
\text { af }(c) \circ \mathcal{T}^{A}+\text { op }(D)
$$

where af $(c)$ is the natural affinor determined by $c \in A, \mathcal{T}^{A}$ the flow operator and op $(D)$ the absolute operator determined by the derivation $D$.

Corollary 3.25. Let $X \in \mathfrak{X}(M)$, any prolongation of $X$ from $M$ to $T^{A} M$ is of the form

$$
\sum_{0 \leq|\alpha| \leq h} a_{\alpha} X^{(\alpha)}+E
$$

where $E$ is an Euler vector on $T^{A} M$ induced by some derivation of $A$ and $a_{0}, a_{\alpha}$ are the real numbers.

Proof. Let $X \in \mathfrak{X}(M)$ and $\tilde{X}$ be a prolongation of $X$ on $T^{A} M$. We have $\widetilde{X}=$ af $(a) \circ \mathcal{T}^{A} X+\mathrm{Op}(D) 0_{M}$, for a some derivation $D$ and $a \in A$. As $a=\sum_{0 \leq|\alpha| \leq h} a_{\alpha} e_{\alpha}$ we obtain

$$
\widetilde{X}=\sum_{0 \leq|\alpha| \leq h} a_{\alpha} X^{(\alpha)}+E
$$

where $E$ is the Euler vector field induced by $D$.

Corollary 3.26. All prolongations of the vector field $X$ from $M$ to $T^{r} M$ are of the form

$$
\sum_{\alpha=0}^{r} a_{\alpha} X^{(\alpha)}+\sum_{\beta=1}^{r} b_{\beta} \xi_{\beta, M}
$$

where $a_{\alpha}, b_{\beta}$ are real numbers.

\section{Homogeneous tensor fields on the WeIl Bundles}

The notion of homogenity for functions on $\mathbb{R}^{n}$ can be extended in an obvious way for functions, vector fields, differential forms, multivector fields on the Weil bundle $T^{A} M$ of a manifold $M$ of dimension $m>0$. In this subsection, we generalize the results of [2] while replacing the tangent bundle of higher order by any Weil bundle. 


\subsection{Homogeneous tensor fields}

Let $M$ be a smooth manifold of dimension $m>0$. We denote by $\xi_{T^{A} M}$ the Euler vector field on the Weil bundle $T^{A} M$. The global flow of $\xi_{T^{A} M}$ is given by the map

$$
F_{t}: T^{A} M \rightarrow T^{A} M, \quad j^{A} g \mapsto j^{A}\left(g_{t}\right)
$$

for any real number $t$.

Definition 4.1. A tensor $\varphi$ on $T^{A} M$ is said to be homogeneous of degree $|\alpha|$ $\left(\alpha \in \mathbb{N}^{k}\right)$ if

$$
F_{t}^{*} \varphi=e^{|\alpha| t} \varphi
$$

for any real number $t$.

Proposition 4.2. A tensor $\varphi$ on $T^{A} M$ is homogeneous of degree $|\alpha|$ if and only if

$$
\mathcal{L}_{\xi_{T} A_{M}} \varphi=|\alpha| \varphi
$$

Proof. Supposing that $\boldsymbol{\varphi}$ is homogeneous tensor fields of degree $|\alpha|$, we have:

$$
\mathcal{L}_{\xi_{T} A_{M}} \boldsymbol{\varphi}=\lim _{t \rightarrow 0}\left(\frac{F_{t}^{*} \boldsymbol{\varphi}-\boldsymbol{\varphi}}{t}\right)=\lim _{t \rightarrow 0}\left(\frac{e^{|\alpha| t}-1}{t}\right) \boldsymbol{\varphi}=|\alpha| \boldsymbol{\varphi} .
$$

Inversely, supposing that $\mathcal{L}_{\xi_{T}{ }_{M}} \varphi=|\alpha| \varphi$, for any $z \in T^{A} M$ the function $X: t \mapsto$ $F_{t}^{*} \boldsymbol{\varphi}(z)$ is the solution of a differential equation $\frac{d u}{d t}=|\alpha| u$ with initial condition $u(0)=\boldsymbol{\varphi}(z)$. Indeed, $X(0)=\boldsymbol{\varphi}(z)$ and $\frac{d}{d t}\left(F_{t}^{*} \boldsymbol{\varphi}\right)=F_{t}^{*} \mathcal{L}_{\xi_{T} A_{M}} \boldsymbol{\varphi}=|\alpha| F_{t}^{*} \boldsymbol{\varphi}$, it follows that $F_{t}^{*} \boldsymbol{\varphi}(z)=e^{|\alpha| t} \boldsymbol{\varphi}(z)$.

Example 4.3. (1) Let $\varphi$ be a tensor field of the type $(0, p)$ on a manifold $M$, for any $|\alpha| \leq h$, the tensor $\varphi^{(\alpha)}$ ( $\alpha$-prolongation of $\varphi$ on $T^{A} M$ ) is a homogeneous tensor field of degree $|\alpha|$.

(2) Let $X$ be a vector field on a manifold $M$. The vector field $X^{(\alpha)}(\alpha-$ prolongation of $X$ on $\left.T^{A} M\right)$ is a homogeneous vector field of degree $-|\alpha|$.

(3) If $f_{1}$ and $f_{2}$ are homogeneous functions of degree $\left|\alpha_{1}\right|$ and $\left|\alpha_{2}\right|$ respectively on $T^{A} M$. Then $f_{1} \cdot f_{2}$ is a homogeneous function of degree $\left|\alpha_{1}\right|+\left|\alpha_{2}\right|$.

Proposition 4.4. If $\varphi_{1}$ and $\varphi_{\mathbf{2}}$ are homogeneous tensor fields on $T^{A} M$ of degree $\left|\alpha_{1}\right|$ and $\left|\alpha_{2}\right|$ respectively. Then, $\boldsymbol{\varphi}_{\mathbf{1}} \otimes \boldsymbol{\varphi}_{\mathbf{2}}$ is homogeneous tensor field on $T^{A} M$ of degree $\left|\alpha_{1}\right|+\left|\alpha_{2}\right|$.

Proof. Given $\varphi_{\mathbf{1}}$ and $\boldsymbol{\varphi}_{\mathbf{2}}$ as in the statement, we have:

$\mathcal{L}_{\xi_{T} A_{M}}\left(\boldsymbol{\varphi}_{\mathbf{1}} \otimes \boldsymbol{\varphi}_{\mathbf{2}}\right)=\left(\mathcal{L}_{\xi_{T} A_{M}} \boldsymbol{\varphi}_{\mathbf{1}}\right) \otimes \boldsymbol{\varphi}_{\mathbf{2}}+\boldsymbol{\varphi}_{\mathbf{1}} \otimes\left(\mathcal{L}_{\xi_{T^{A}{ }_{M}}} \boldsymbol{\varphi}_{\mathbf{2}}\right)=\left|\alpha_{1}\right| \boldsymbol{\varphi}_{\mathbf{1}} \otimes \boldsymbol{\varphi}_{\mathbf{2}}+\left|\alpha_{2}\right| \boldsymbol{\varphi}_{\mathbf{1}} \otimes \boldsymbol{\varphi}_{\mathbf{2}}$. It follows that $\varphi_{1} \otimes \varphi_{2}$ is a homogeneous tensor field of degree $\left|\alpha_{1}\right|+\left|\alpha_{2}\right|$.

Corollary 4.5. If $X_{1}$ and $X_{2}$ are homogeneous vector fields on $T^{A} M$ of degree $\left|\alpha_{1}\right|$ and $\left|\alpha_{2}\right|$ respectively. Then $\left[X_{1}, X_{2}\right]$ is homogeneous vector field on $T^{A} M$ of degree $\left|\alpha_{1}\right|+\left|\alpha_{2}\right|$.

Proof. Let $X_{1}$ and $X_{2}$ be homogeneous vector fields on $T^{A} M$ of degree $\left|\alpha_{1}\right|$ and $\left|\alpha_{2}\right|$. Using the Jacobi identity, we have

$$
\mathcal{L}_{\xi_{T} A_{M}}\left[X_{1}, X_{2}\right]=\left[X_{1}, \mathcal{L}_{\xi_{T} A_{M}} X_{2}\right]+\left[\mathcal{L}_{\xi_{T} A_{M}} X_{1}, X_{2}\right]=\left(\left|\alpha_{1}\right|+\left|\alpha_{2}\right|\right)\left[X_{1}, X_{2}\right] .
$$


Therefore, $\left[X_{1}, X_{2}\right]$ is a homogeneous vector field of degree $\left|\alpha_{1}\right|+\left|\alpha_{2}\right|$.

Corollary 4.6. Let $X$ be a homogeneous vector field of degree $|\alpha|$ and $f$ a homogeneous function of degree $|\beta|$. Then $X(f)$ is a homogeneous function of degree $|\alpha|-|\beta|$.

Proof. We have

$$
\mathcal{L}_{\xi_{T} A_{M}} X(f)=\left(\mathcal{L}_{\xi_{T} A_{M}} X\right)(f)-|\beta| X(f)=|\alpha| X(f)-|\beta| X(f)
$$

and, therefore, $X(f)$ is a homogeneous function of degree $|\alpha|-|\beta|$.

\subsection{Particular case of the differential forms}

Proposition 4.7. (1) Let $\omega_{1}$ and $\omega_{2}$ be homogeneous forms of degree $\left|\alpha_{1}\right|$ and $\left|\alpha_{2}\right|$ respectively. Then $\omega_{1} \wedge \omega_{2}$ is homogeneous form of degree $\left|\alpha_{1}\right|+$ $\left|\alpha_{2}\right|$.

(2) Let $\omega$ be a homogeneous $p$-form of degree $|\alpha|$ and $X_{1}, \cdots, X_{p}$, p homogeneous vector fields of degree $\left|\alpha_{1}\right|, \cdots,\left|\alpha_{p}\right|$. Then $\omega\left(X_{1}, \cdots, X_{p}\right)$ is a homogeneous function of degree $|\alpha|+\left|\alpha_{1}\right|+\cdots+\left|\alpha_{p}\right|$.

Proof. We know that

$$
\begin{aligned}
\mathcal{L}_{\xi_{T} A_{M}}\left(\omega\left(X_{1}, \cdots, X_{p}\right)\right)=\mathcal{L}_{\xi_{T} A_{M}} \omega\left(X_{1}, \cdots, X_{p}\right) & \\
& +\sum_{i=1}^{n} \omega\left(X_{1}, \cdots, \mathcal{L}_{\xi_{T} A_{M}} X_{i}, \cdots, X_{p}\right) .
\end{aligned}
$$

As $\mathcal{L}_{\xi_{T} A_{M}} \omega=|\alpha| \omega$ and $\mathcal{L}_{\xi_{T} A_{M}} X_{i}=\left|\alpha_{i}\right| X_{i}$ for any $i \leq p$, we have

$$
=|\alpha| \omega\left(X_{1}, \cdots, X_{p}\right)+\left(\left|\alpha_{1}\right|+\cdots+\left|\alpha_{p}\right|\right) \omega\left(X_{1}, \cdots, X_{p}\right) .
$$

So, we obtain the result.

Remark 4.8. Let $\left(x^{i}, x_{\alpha}^{i}\right)_{\alpha \in B_{A}}$ be an adapted local coordinate system of $T^{A} M$. The local expression of the Pfaff form $\omega$ on $T^{A} M$ is given by

$$
\omega=a_{0}^{i} d x^{i}+\sum_{\alpha \in B_{A}} a_{i}^{\alpha} d x_{\alpha}^{i}
$$

and we have

$$
\mathcal{L}_{\xi_{T^{A} M}} \omega=\sum_{\alpha \in B_{A}} \xi_{T^{A} M}\left(a_{i}^{\alpha}\right) d x_{\alpha}^{i}+a_{i}^{\alpha} \xi_{T^{A} M}\left(d x_{\alpha}^{i}\right)
$$

Now, $\xi_{T^{A} M}=\sum_{\beta \in B_{A}}|\beta| x_{\beta}^{j} \frac{\partial}{\partial x_{\beta}^{j}}$ and $\xi_{T^{A} M}\left(d x_{\alpha}^{i}\right)=|\alpha| d x_{\alpha}^{i}$ and we have

$$
\mathcal{L}_{\xi_{T_{A} A_{M}}} \omega=\sum_{\alpha, \beta \in B_{A}}|\beta| x_{\beta}^{j} \frac{\partial a_{i}^{\alpha}}{\partial x_{\beta}^{j}} d x_{\alpha}^{i}+|\alpha| a_{i}^{\alpha} d x_{\alpha}^{i}=\sum_{\alpha \in B_{A}}\left(\sum_{\beta \in B_{A}}|\beta| x_{\beta}^{j} \frac{\partial a_{i}^{\alpha}}{\partial x_{\beta}^{j}}+|\alpha| a_{i}^{\alpha}\right) d x_{\alpha}^{i} .
$$

If $\omega$ is homogeneous of degree $|\gamma|$, then we have $a_{0}^{i}=0$ and

$$
\sum_{\beta \in B_{A}}|\beta| x_{\beta}^{j} \frac{\partial a_{i}^{\alpha}}{\partial x_{\beta}^{j}}+|\alpha| a_{i}^{\alpha}=|\gamma| a_{i}^{\alpha} .
$$


Thus, we obtain

$$
\sum_{\beta \in B_{A}}|\beta| x_{\beta}^{j} \frac{\partial a_{i}^{\alpha}}{\partial x_{\beta}^{j}}=(|\gamma|-|\alpha|) a_{i}^{\alpha} .
$$

It follows that, for each $0<i \leq m$ and $\alpha \in B_{A}$, the function $a_{i}^{\alpha}$ is homogeneous of degree $|\gamma|-|\alpha|$.

Proposition 4.9. Let $\omega$ be a homogeneous p-form on $T^{A} M$ of degree $|\alpha|$. Then $d \omega, i_{\xi_{T} A_{M}} \omega$ are homogeneous of degree $|\alpha|$.

Proof. In the first case,

$$
\mathcal{L}_{\xi_{T} A_{M}}(d \omega)=d\left(\mathcal{L}_{\xi_{T^{A}}{ }_{M}} \omega\right)=|\alpha| d \omega
$$

By the same argument,

$$
\mathcal{L}_{\xi_{T} A_{M}}\left(i_{\xi_{T} A_{M}} \omega\right)=i_{\xi_{T^{A} A_{M}}} d\left(i_{\xi_{T^{A} A_{M}}} \omega\right)=i_{\xi_{T^{A} M_{M}}}\left(\mathcal{L}_{\xi_{T^{A}{ }_{M}}} \omega\right)=|\alpha|\left(i_{\xi_{T^{A} A_{M}}} \omega\right) .
$$

Therefore $i_{\xi_{T} A_{M}} \omega$ and $d \omega$ are homogeneous of degree $|\alpha|$.

\subsection{Case of multivector fields}

Proposition 4.10. (1) Let $\pi_{1}$ and $\pi_{2}$ be homogeneous multivector fields on $T^{A} M$ of degree $\left|\alpha_{1}\right|$ and $\left|\alpha_{2}\right|$ respectively. Then, $\pi_{1} \wedge \pi_{2}$ is homogeneous multivector field of degree $\left|\alpha_{1}\right|+\left|\alpha_{2}\right|$.

(2) If $\pi=X_{1} \wedge \cdots \wedge X_{p}$ is a simple multivector field on $T^{A} M$, where $X_{1}, \cdots, X_{p}$ are $p$ homogeneous vector fields of degree $\left|\alpha_{1}\right|, \cdots,\left|\alpha_{p}\right|$. Then, $\pi$ is a homogeneous multivector field on $T^{A} M$ of degree $\left|\alpha_{1}\right|+\cdots+\left|\alpha_{p}\right|$.

(3) Let $\pi_{1}$ and $\pi_{2}$ be homogeneous multivector fields on $T^{A} M$ of degree $\left|\alpha_{1}\right|$ and $\left|\alpha_{2}\right|$ respectively. Then, $\left[\pi_{1}, \pi_{2}\right]$ is a homogeneous multivector field of degree $\left|\alpha_{1}\right|+\left|\alpha_{2}\right|$.

Proof. Let $\pi_{1} \in \mathfrak{X}^{p}\left(T^{A} M\right)$ and $\pi_{2} \in \mathfrak{X}^{q}\left(T^{A} M\right)$, we have:

$$
\begin{aligned}
\mathcal{L}_{\xi_{T} A_{M}}\left[\pi_{1}, \pi_{2}\right] & =-(-1)^{(p-1)(q-1)}\left[\pi_{2}, \mathcal{L}_{\xi_{T^{A}}{ }_{M}} \pi_{1}\right]+\left[\pi_{1}, \mathcal{L}_{\xi_{T} A_{M}} \pi_{2}\right] \\
& =-(-1)^{(p-1)(q-1)}\left|\alpha_{1}\right|\left[\pi_{2}, \pi_{1}\right]+\left|\alpha_{2}\right|\left[\pi_{1}, \pi_{2}\right] .
\end{aligned}
$$

We deduce that $\mathcal{L}_{\xi_{T^{A} M_{M}}}\left[\pi_{1}, \pi_{2}\right]=\left(\left|\alpha_{1}\right|+\left|\alpha_{2}\right|\right)\left[\pi_{1}, \pi_{2}\right]$.

Proposition 4.11. Let $\pi \in \mathfrak{X}^{p}\left(T^{A} M\right)$ be a homogeneous multivector field of degree $|\alpha|$. For any $p$ homogeneous functions $f_{1}, \cdots, f_{p}$ on $T^{A} M$ of degree $\left|\alpha_{1}\right|, \cdots,\left|\alpha_{p}\right|$ the function $\pi\left(d f_{1}, \cdots, d f_{p}\right)$ is homogeneous of degree $|\alpha|+\left|\alpha_{1}\right|+$ $\cdots+\left|\alpha_{p}\right|$.

Proof. We have,

$$
\begin{aligned}
& \mathcal{L}_{\xi_{T^{A} M}}\left(\pi\left(d f_{1}, \cdots, d f_{p}\right)\right) \\
= & \mathcal{L}_{\xi_{T^{A} A_{M}}} \pi\left(d f_{1}, \cdots, d f_{p}\right)+\sum_{i=1}^{p} \pi\left(d f_{1}, \cdots \mathcal{L}_{\xi_{T^{A} M_{M}}} d f_{i}, \cdots, d f_{p}\right) \\
= & |\alpha| \pi\left(d f_{1}, \cdots, d f_{p}\right)+\sum_{i=1}^{n}\left|\alpha_{i}\right| \pi\left(d f_{1}, \cdots, d f_{p}\right) .
\end{aligned}
$$


We deduce that

$$
\mathcal{L}_{\xi_{T^{A} M}}\left(\pi\left(d f_{1}, \cdots, d f_{p}\right)\right)=\left(|\alpha|+\left|\alpha_{1}\right|+\cdots+\left|\alpha_{p}\right|\right) \pi\left(d f_{1}, \cdots, d f_{p}\right) .
$$

Let $M$ be a smooth $m$-dimensional manifold. A Poisson structure on $M$ is a $\mathbb{R}$-bilinear Lie bracket $\{\cdot, \cdot\}$ on $C^{\infty}(M)$ satisfying the Leibnitz rule

$$
\{f, g h\}=\{f, g\} h+g\{f, h\}, \quad \forall f, g, h \in C^{\infty}(M) .
$$

It follows from (4.1) that there exists a bivector field $w \in \mathfrak{X}^{2}(M)$ such that

$$
\{f, g\}_{w}=w(d f, d g) .
$$

The Jacobi identity for $\{\cdot, \cdot\}_{w}$ is equivalent to the Poisson condition $[w, w]=0$, where $[\cdot, \cdot]$ is the Schouten-Nijenhuis bracket. In this case, one says that the bivector field $w$ defines the Poisson structure on $M$.

Proposition 4.12. Let $\pi$ be a Poisson bivector on $T^{A} M$ homogeneous of degree $|\alpha|$. For any homogeneous function $f$ of degree $|\beta|$, the hamiltonian vector field $X_{f}$ is a homogeneous vector field of degree $|\alpha|+|\beta|$.

Proof. Let $g \in C^{\infty}(M)$ and $|\gamma| \leq h$, we have:

$$
\begin{gathered}
\mathcal{L}_{\xi_{T^{A} M}} X_{f}\left(g^{(\gamma)}\right)=\left[\xi_{T^{A} M}, X_{f}\right]\left(g^{(\gamma)}\right)=\xi_{T^{A} M}\left(\pi\left(d f, d g^{(\gamma)}\right)\right)-|\gamma| X_{f}\left(g^{(\gamma)}\right) \\
=(|\alpha|+|\beta|+|\gamma|) \pi\left(d f, d g^{(\gamma)}\right)-|\gamma| X_{f}\left(g^{(\gamma)}\right)=(|\alpha|+|\beta|) X_{f}\left(g^{(\gamma)}\right) .
\end{gathered}
$$

Therefore, $\mathcal{L}_{\xi_{T} A_{M}} X_{f}=(|\alpha|+|\beta|) X_{f}$.

Corollary 4.13. Let $\pi$ be a Poisson bivector on $T^{A} M$ homogeneous of degree $|\alpha|$. If $f_{1}$ and $f_{2}$ are homogeneous functions of degree $\left|\alpha_{1}\right|$ and $\left|\alpha_{2}\right|$ respectively. Then, the function $\left\{f_{1}, f_{2}\right\}$ is homogeneous of degree $|\alpha|+\left|\alpha_{1}\right|+\left|\alpha_{2}\right|$.

5. Homogeneous properties of Euler vector fields on SOME GEOMETRIC STRUCTURES

In the sequel, by $\langle\cdot, \cdot\rangle_{M}$ we denote the canonical pairing $T M \times_{M} T^{*} M \rightarrow \mathbb{R}$.

\subsection{Case of the tangent lifts of Poisson manifolds}

For any manifold $M$ of dimension $m \geq 1$, there is a canonical diffeomorphism (see $[1,3,5])$

$$
\kappa_{M}^{r}: T^{r} T M \rightarrow T T^{r} M
$$

which is an isomorphism of vector bundles from

$$
T^{r}\left(\pi_{M}\right): T^{r} T M \rightarrow T^{r} M \quad \text { to } \quad \pi_{T^{r} M}: T T^{r} M \rightarrow T^{r} M .
$$

It is called the canonical isomorphism of flow associated to the bundle functor $T^{r}$. Consider the linear form $\tau_{r}$ on $J_{0}^{r}(\mathbb{R}, \mathbb{R})$ defined by $\tau_{r}\left(j_{0}^{r} g\right)=\left.\frac{1}{r !} \frac{d^{r} g}{d t^{r}}(t)\right|_{t=0}$ and the canonical map

$$
\alpha_{M}^{r}: T^{*} T^{r} M \rightarrow T^{r} T^{*} M
$$

which is an isomorphism of vector bundles

$$
\pi_{T^{r} M}^{*}: T^{*} T^{r} M \rightarrow T^{r} M \quad \text { and } \quad T^{r}\left(\pi_{M}^{*}\right): T^{r} T^{*} M \rightarrow T^{r} M
$$


such that, for any $\left(u, u^{*}\right) \in T^{r} T M \oplus T^{*} T^{r} M$,

$$
\left\langle\kappa_{M}^{r}(u), u^{*}\right\rangle_{T^{r} M}=\left\langle u, \alpha_{M}^{r}\left(u^{*}\right)\right\rangle_{T^{r} M}^{\prime}
$$

where $\langle\cdot, \cdot\rangle_{T^{r} M}^{\prime}=\tau_{r} \circ T^{r}\left(\langle\cdot, \cdot\rangle_{M}\right)$ (see [1]). We denote by $\varepsilon_{M}^{r}$ the inverse of $\alpha_{M}^{r}$.

Let $\left(x^{1}, \cdots, x^{m}\right)$ be a local coordinate system of $M$, we introduce the coordinates $\left(x^{i}, p_{j}\right)$ in $T^{*} M,\left(x^{i}, p_{j}, x_{\beta}^{i}, p_{j}^{\beta}\right)$ in $T^{r} T^{*} M$ and $\left(x^{i}, x_{\beta}^{i}, \pi_{j}, \pi_{j}^{\beta}\right)$ in $T^{*} T^{r} M$. We have:

$$
\alpha_{M}^{r}\left(x^{i}, \pi_{j}, x_{\beta}^{i}, \pi_{j}^{\beta}\right)=\left(x^{i}, x_{\beta}^{i}, p_{j}, p_{j}^{\beta}\right), \text { with }\left\{\begin{array}{l}
p_{j}=\pi_{j}^{r} \\
p_{j}^{\beta}=\pi_{j}^{r-\beta}
\end{array} .\right.
$$

Let $(M, w)$ be a Poisson manifold. The complete lift of higher order of $w$ in the sense of [6] and denoted by $w^{(c)}$ is a Poisson bivector field on $T^{r} M$ since the Poisson condition $\left[w^{(c)}, w^{(c)}\right]=0$, is satisfied. Denoting by $\sharp_{w}: T^{*} M \rightarrow T M$ the anchor map induced by $w$, we have

$$
\sharp_{w^{(c)}}=\alpha_{M}^{r} \circ T^{r}\left(\sharp_{w}\right) \circ \kappa_{M}^{r} .
$$

Let $\left(x^{1}, \cdots, x^{m}\right)$ be a local coordinate system of $M$ such that $w=w^{i j} \frac{\partial}{\partial x^{i}} \wedge \frac{\partial}{\partial x^{j}}$, we have

$$
w^{(c)}=\left(w^{i j}\right)^{(\alpha+\beta-r)} \frac{\partial}{\partial x_{\alpha}^{i}} \wedge \frac{\partial}{\partial x_{\beta}^{j}} .
$$

In [6], we have shown that, for any $f, g \in C^{\infty}(M)$, we have:

$$
\left\{f^{(\alpha)}, g^{(\beta)}\right\}_{w^{(c)}}=\{f, g\}_{w}^{(\alpha+\beta-r)}
$$

with $0 \leq \alpha, \beta \leq r$.

Definition 5.1. The Poisson manifold $(M, w)$ is said homogeneous related to a vector field $X \in \mathfrak{X}(M)$ if $\mathcal{L}_{X} w=-w$.

Theorem 5.2. The Poisson manifold $\left(T^{r} M, w^{(c)}\right)$ is a homogeneous Poisson manifold related to $\frac{1}{r} \cdot \xi_{T^{r} M}$. More precisely,

$$
\left[\xi_{T^{r} M}, w^{(c)}\right]=-r w^{(c)} .
$$

Proof. For any $f, g \in C^{\infty}(M)$, we have

$$
\begin{aligned}
{\left[\xi_{T^{r} M}, w^{(c)}\right]\left(d f^{(\alpha)} \wedge d g^{(\beta)}\right)=} & \left\langle\xi_{T^{r} M}, d\left(i_{w^{(c)}}\left(d f^{(\alpha)} \wedge d g^{(\beta)}\right)\right)\right\rangle_{T^{r} M} \\
& -\left\langle w^{(c)}, d\left(\left(i_{\xi_{T^{r} M}} d f^{(\alpha)}\right) \wedge d g^{(\beta)}\right)\right\rangle_{T^{r} M}
\end{aligned}
$$

Therefore,

$$
\begin{aligned}
& {\left[\xi_{T^{r} M}, w^{(c)}\right]\left(d f^{(\alpha)} \wedge d g^{(\beta)}\right)} \\
& =-\left\langle w^{(c)}, \alpha d f^{(\alpha)} \wedge d g^{(\beta)}+\beta d f^{(\alpha)} \wedge d g^{(\beta)}\right\rangle_{T^{r} M}+\xi_{T^{r} M}\left(\{f, g\}_{w}^{(\alpha+\beta-r)}\right) \\
& =(\alpha+\beta-r)\{f, g\}_{w}^{(\alpha+\beta-r)}-\left\langle w^{(c)},(\alpha+\beta) d f^{(\alpha)} \wedge d g^{(\beta)}\right\rangle \\
& =(\alpha+\beta-r)\{f, g\}_{w}^{(\alpha+\beta-r)}-(\alpha+\beta)\{f, g\}_{w}^{(\alpha+\beta-r)} .
\end{aligned}
$$

We deduce that

$$
\left[\xi_{T^{r} M}, w^{(c)}\right]\left(d f^{(\alpha)} \wedge d g^{(\beta)}\right)=-r\{f, g\}_{w}^{(\alpha+\beta-r)}=-r w^{(c)}\left(d f^{(\alpha)} \wedge d g^{(\beta)}\right) .
$$

Thus, $\left[\xi_{T^{r} M}, w^{(c)}\right]=-r w^{(c)}$.

Remark 5.3. For $r=1$, we obtain the result established by I. Vaisman in [9]. 


\subsection{Tangent Dirac structures of higher order}

Let $M$ be a smooth manifold of dimension $m \geq 1$. We recall that, an almost Dirac structure on $M$ is a subbundle of $L \subset T M \oplus T^{*} M$ of rank $m$ which is maximally isotrope related to the canonical pairing on $T M \oplus T^{*} M$ defined by

$$
\langle X \oplus \omega, Y \oplus \varpi\rangle_{+}=\frac{1}{2}\left(\langle Y, \omega\rangle_{M}+\langle X, \varpi\rangle_{M}\right) .
$$

We put

$$
\langle X \oplus \omega, Y \oplus \varpi\rangle_{-}=\frac{1}{2}\left(\langle Y, \omega\rangle_{M}-\langle X, \varpi\rangle_{M}\right) .
$$

If the space of local sections of $L$ denoted by $\Gamma(L)$ is closed under the bracket,

$$
[X \oplus \omega, Y \oplus \varpi]=[X, Y] \oplus\left(\mathcal{L}_{X} \varpi-\mathcal{L}_{Y} \omega+d\left(\langle X \oplus \omega, Y \oplus \varpi\rangle_{-}\right)\right)
$$

we say that $L$ is a Dirac structure on $M$.

Definition 5.4. A Dirac structure $L$ on $M$ is called a homogeneous Dirac structure related to a vector field $Z$ on $M$ if, for any $(X, \omega) \in \Gamma(L),\left([Z, X]+X, \mathcal{L}_{Z} \omega\right) \in$ $\Gamma(L)$.

Let $L \subset T M \oplus T^{*} M$ be a Dirac structure, we put

$$
\mathcal{T}^{r} L=\left(\kappa_{M}^{r} \oplus \varepsilon_{M}^{r}\right)\left(T^{r} L\right) \subset T T^{r} M \oplus T^{*} T^{r} M .
$$

The subbundle $\mathcal{T}^{r} L \subset T T^{r} M \oplus T^{*} T^{r} M$ is a Dirac structure on $T^{r} M$ (see [5]). It is called tangent Dirac structure of higher order.

Lemma 5.5. Let $L \subset T M \oplus T^{*} M$ be a Dirac structure on $M$. We have:

$$
X^{(\beta)} \oplus w^{(r-\beta)} \in \Gamma\left(\mathcal{T}^{r} L\right)
$$

for any $X \oplus w \in \Gamma(L)$ and $\beta=0, \cdots, r$.

Proof. See [7].

Theorem 5.6. The Dirac structure $\mathcal{T}^{r} L$ on $T^{r} M$ is a homogeneous Dirac structure related to Euler vector field $\frac{\xi_{T^{r} M}}{r}$.

Proof. We recall that the space of sections of $\mathcal{T}^{r} L$ is generated by the space

$$
\left\{X^{(\alpha)} \oplus w^{(r-\alpha)}, X \oplus w \in \Gamma(L) \text { and } \alpha=0, \cdots, r\right\} .
$$

For each section $\left(X_{1}, w_{1}\right)$ of $L$, using the equalities

$$
\left\{\begin{array}{ccc}
{\left[\frac{\xi_{T} r_{M}}{r}, X_{1}^{(\alpha)}\right]} & =-\frac{\alpha}{r} X_{1}^{(\alpha)} \\
\mathcal{L}_{\frac{\xi_{T} r_{M}}{r}} w_{1}^{(r-\alpha)} & =\left(1-\frac{\alpha}{r}\right) w_{1}^{(r-\alpha)}
\end{array}\right.
$$

we have

$$
\left(\left[\frac{\xi_{T^{r} M}}{r}, X_{1}^{(\alpha)}\right]+X_{1}^{(\alpha)}\right) \oplus \mathcal{L}_{\frac{\xi_{T^{r} M}}{r}} w_{1}^{(r-\alpha)}=X_{1}^{(\alpha)} \oplus w_{1}^{(r-\alpha)}-\frac{\alpha}{r}\left(X_{1}^{(\alpha)} \oplus w_{1}^{(r-\alpha)}\right)
$$

$X_{1}^{(\alpha)} \oplus w_{1}^{(r-\alpha)}$ is a section of $\mathcal{T}^{r} L$, which means that

$$
\left(\left[\frac{\xi_{T^{r} M}}{r}, X_{1}^{(\alpha)}\right]+X_{1}^{(\alpha)}\right) \oplus \mathcal{L}_{\frac{\xi_{T^{r} M}}{r}} w_{1}^{(r-\alpha)}=\left(1-\frac{\alpha}{r}\right)\left(X_{1}^{(\alpha)} \oplus w_{1}^{(r-\alpha)}\right)
$$


is a section of $\mathcal{T}^{r} L$. Thus, $\mathcal{T}^{r} L$ is a homogeneous Dirac structure related to the vector field $\frac{\xi_{T^{r} M}}{r}$.

\section{REFERENCES}

[1] F. Cantrijn, M. Crampin, W. Sarlet and D. Saunders, The canonical isomorphism between $T^{k} T^{*}$ and $T^{*} T^{k}$, C. R. Acad. Sci., Paris, Sr. II 309 (1989), 1509-1514.

[2] M. de León and P. R. Rodrigues, Generalized Classical Mechanics and Field Theory, NorthHolland Mathematics Studies 112, Elsevier Science Publishers, 1985.

[3] J. Gancarzewicz, W. Mikulski and Z. Pogoda, Lifts of some tensor fields and connections to product preserving functors, Nagoya Math. J. 135 (1994), 41 pp.

[4] I. Kolář, P. Michor and J. Slovák, Natural Operations in Differential Geometry, SpringerVerlag, Berlin, 1993.

[5] P. M. Kouotchop Wamba, A. Ntyam and J. Wouafo Kamga, Tangent Dirac structures of higher order, Arch. Math., Brno 47 (2011), 17-22.

[6] P. M. Kouotchop Wamba, A. Ntyam and J. Wouafo Kamga, Tangent lifts of higher order of multivector fields and applications, J. Math. Sci. Adv. Appl. 15 (2012), 13-36.

[7] P. M. Kouotchop Wamba, A. Ntyam and J. Wouafo Kamga, Some properties of tangent Dirac structures of higher order, Arch. Math., Brno 48 (2012), 233-241.

[8] G. Mitric and I. Vaisman, Poisson structures on tangent bundles,Differ. Geom. Appl. 18 (2003), 207-228.

[9] I. Vaisman, Tangent Dirac structures and submanifolds, Int. J. Geom. Methods Mod. Phys. 2 (2005), 759-775.

P. M. Kouotchop Wamba, Department of Mathematics, University of Yaounde I, Higher Teacher Training College, PO.BOX 47 Yaounde, Cameroon

e-mail: wambapm@yahoo.fr 